\title{
A brief review of microRNA and its role in PRRSV infection and replication
}

\author{
Xuekun GUO, Wenhai FENG (ه) \\ State Key Laboratory of Agrobiotechnology, Ministry of Agriculture Key Laboratory of Soil Microbiology, Department of Microbiology and \\ Immunology, College of Biological Sciences, China Agricultural University, Beijing 100193, China
}

\begin{abstract}
Porcine reproductive and respiratory syndrome virus (PRRSV), a single-stranded RNA virus, mainly infects cells of monocyte/macrophage lineage. Recently, host microRNAs were shown to be capable of modulating PRRSV infection and replication by multiple ways such as targeting viral genomic RNA, targeting viral receptor and inducing antiviral response. MicroRNAs are small RNAs and have emerged as important regulators of virus-host cell interactions. In this review, we discuss the identified functions of host microRNAs in relation to PRRSV infection and propose that cellular microRNAs may have a substantial effect on cell or tissue tropism of PRRSV.
\end{abstract}

Keywords porcine reproductive and respiratory syndrome virus (PRRSV), microRNA, antiviral, viral tropism

\section{Introduction}

Porcine reproductive and respiratory syndrome virus (PRRSV) was identified in Europe and the United States in the early 1990s and has become an important pathogen in the swine industry worldwide. Pigs of all ages can be infected by PRRSV, which causes severe disease characterized by reproductive failure in pregnant sows, and respiratory problems and persistent infection in piglets [1]. A typical highly-pathogenic PRRSV (HP-PRRSV) emerged in China in 2006 and is characterized by high fever, high morbidity and high mortality in pigs of all ages. Vaccines have been developed, but they fail to provide sustainable protection, in particular against highly pathogenic strains.

MicroRNAs (miRNAs) are endogenous about 21-23 nt RNA molecules that have emerged as critical gene regulators in animals and plants [2,3]. It is believed that in mammals miRNAs control the activity of approximately

Received March 28, 2014; accepted April 20, 2014

Correspondence: whfeng@cau.edu.cn
$50 \%$ of all protein-coding genes [4]. Increasing evidence indicates that miRNAs can participate in the regulation of almost every cellular activity and their abnormal expression is associated with many human diseases. Recently, several studies have demonstrated that miRNAs can regulate PRRSV infection and replication [5-8]. Here we discuss the current understanding of the role of miRNA in PRRSV infection and hypothesize that cellular miRNAs may contribute significantly to regulating PRRSV infection.

\section{Animal miRNA biogenesis and function}

The general miRNA biogenesis pathway is illustrated in Fig. 1, and this topic has been comprehensively reviewed elsewhere [4]. Briefly, miRNAs are first processed from primary transcripts of miRNAs (pri-miRNAs), which can be transcribed by RNA polymerase II (RNAPII) either from independent genes or introns of protein-coding genes [3,9]. A single pri-miRNA which folds into hairpins may contain a cluster of distinct miRNAs or only a single miRNA. The pri-miRNAs act as substrates for two members of the RNase III family of enzymes, Drosha and Dicer. The nuclear endoribonuclease Drosha cleaves a pri-miRNA to generate an approximately $70-80$ nt hairpincontaining precursor miRNA (pre-miRNA) [10], which will be exported to the cytoplasm by exportin-5. Cytoplasmic pre-miRNAs are then recognized and processed into an approximately 22 bp miRNA/miRNA* duplex by Dicer. One strand of this duplex (referred to as the guide strand or a mature miRNA) is then ultimately incorporated into the miRNA-induced silencing complex (miRISC), whereas the other strand (passenger or miRNA*) is released and degraded. This miRISC-loaded guide strand is responsible for binding to target mRNAs in a sequence-dependent manner and induces their translational inhibition or deadenylation and degradation [11].

The mechanism by which miRNAs regulate target gene 


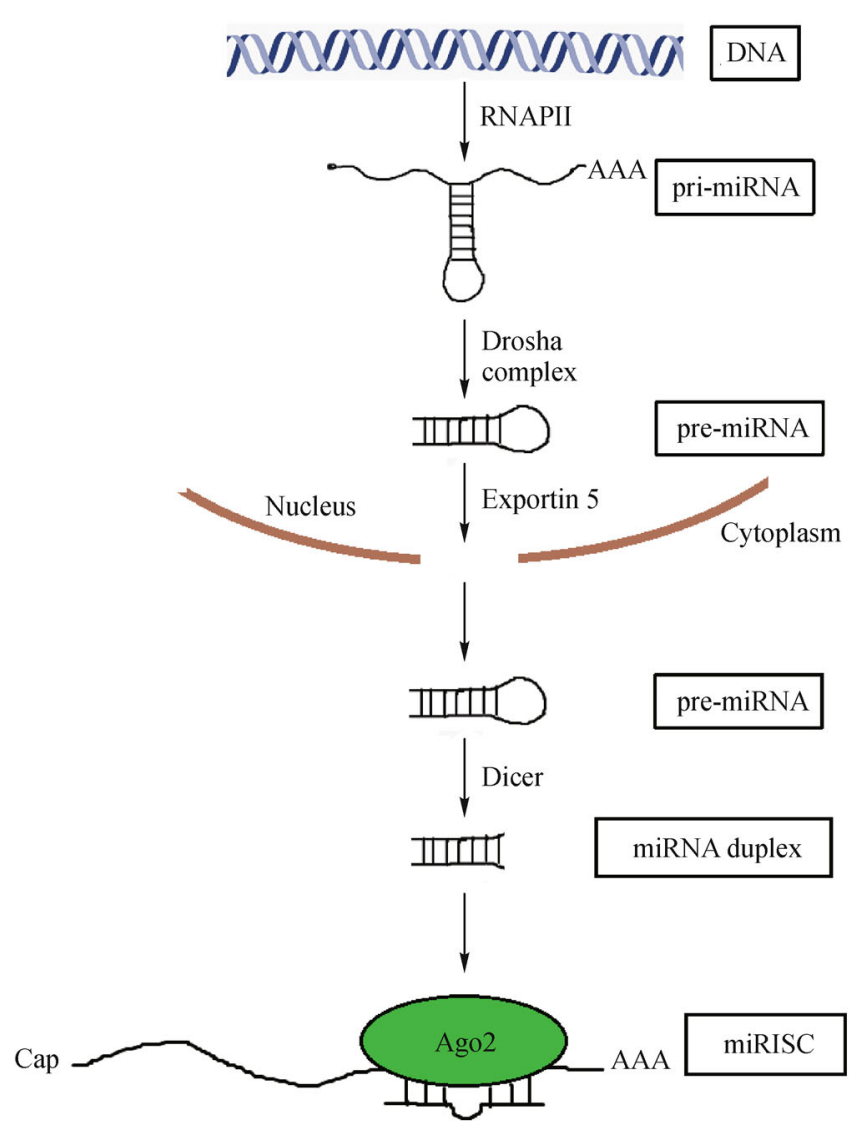

Translational inhibition, mRNA deadenylation and degradation

Fig. 1 Biogenesis and function of animal miRNAs. For simplicity, not all factors involved in miRNA processing are shown. Ago2, Argonaute 2 which directly interact with miRNAs in the miRISC.

expression has been a controversial subject, as it has been found that miRNAs could result in target mRNA destabilization, translational repression, and even activation of gene expression, respectively [12]. Most animal miRNAs pair imperfectly with target sites in the 3' UTR of target mRNAs to inhibit gene expression. In most cases, mRNA targeting involves perfect pairing between a target and nucleotides 2-7 (or 8) of the miRNA, a common motif, which is called the seed region. In some experimental contexts, seed pairing has been shown to be both essential and sufficient for regulation by the miRNA pathway [13]. Sometimes imperfect pairing between the $5^{\prime}$ end of the miRNA and a target mRNA can be compensated for by extensive $3^{\prime}$ end interactions, which has been found in the case of a let-7 miRNA target site in the lin-41 3' UTR in Caenorhabditis elegans [14-16]. Recently, centered sites have been described as a class of miRNA target sites that lack both perfect seed pairing and 3' compensatory pairing and instead have 11-12 continuous Watson-Crick pairs to the center of the miRNA [17]. Besides, there are also some examples of functional miRNA target sites that do not match any of the above described patterns $[18,19]$. The flexible pairing patterns in animal miRNA-targeting suggest that factors beyond base-pairing are involved in miRNA-mRNA interactions in vivo.

\section{The role of animal miRNAs in pathogen infections}

Microorganisms that invade a vertebrate host are initially recognized by the innate immune system, which is the first line of host defense against pathogens. The innate immune response involves several immune cells including macrophages, granulocyte and dendritic cells, which are responsible for recognizing specific molecules of pathogens via a limited number of germline-encoded patternrecognition receptors [20]. The specific molecules or components from pathogens, known as pathogen-associated molecular patterns (PAMPs), are usually essential for the survival of the pathogens and are therefore hard for the pathogens to change. In many cases, Toll-like patternrecognition receptors (TLRs) can recognize invasions of a variety of pathogens. For example, Toll-like receptor 3 (TLR3) and TLR7 can recognize microbial dsRNA and ssRNA, respectively. TLR3 and TLR7 are therefore usually associated with recognition of viruses, while TLR4 is mainly responsible for recognizing PAMPs of bacteria [21]. After the engagement of TLRs by their PAMPs or ligands, specific biological responses are triggered by individual TLRs. The specific responses include myeloid differentiation factor 88 (MyD88)-dependent signaling pathways, which activate the induction of proinflammatory cytokines, and Toll-IL-1 receptor domain-containing adaptor inducing IFN- $\beta$ (TRIF)-dependent signaling pathways, which drive the induction of type I interferon as well as proinflammatory cytokines [20]. These antiviral cytokines and proinflammatory cytokines are required for inducing host innate and adaptive immune response to destroy pathogens.

miRNAs have recently emerged as key gene-regulators and have been shown to play an important role in innate immune response to infections [22,23]. It is well known that TLRs signaling can induce hundreds of TLRresponsive coding genes. As with TLR-responsive coding genes, miRNAs can be induced at the early or late stage of responses [22]. For example, miR-155 is highly induced 2 $\mathrm{h}$ after stimulation, whereas miR-21 is induced at later times. Interestingly, miR-155 and miR-146a are upregulated by bacterial or viral ligands, and this upregulation is dependent on nuclear factor- $\mathrm{\kappa B}(\mathrm{NF}-\mathrm{\kappa} B)$ activity, which is the downstream of TLR signaling [24-26]. In addition to these two miRNAs, many other miRNAs in different immune cells have been reported in succession [22,23]. Among all the immune-responsive miRNAs, miR-155, miR-146a, and miR-21 have been particularly well characterized. It has been shown that miR-155 can positively regulate inflammatory cytokines by targeting 
SH2-domain-containing inositol-5-phosphatase 1, which is a key negative regulator of phosphoinositide 3-kinase and the downstream activated form of the serine/threonine kinase (AKT) signaling [27]. Similarly, miR-155 also positively regulates type I interferons by targeting suppressor of cytokine signaling 1 , which is a negative regulator of TLR signaling [28,29]. Unlike miR-155, miR$146 \mathrm{a}$ is a negative regulator of immune response, because it can repress TLR signal transduction by targeting TNFRassociated factor 6 (TRAF6) and IL-1R-associated kinase 1 (IRAK1) [25]. Interestingly, miR-146a can be induced during viral infection, which is dependent on nuclear factor kappa-light-chain-enhancer of activated B cells (NF- $\mathrm{B}$ ) activation, suggesting the existence of a negative feedback loop [30]. Another miRNA, miR-21, induced in various immune situations, can also negatively regulate immune response by targeting programmed cell death protein 4 (PDCD4), MyD88, and interleukin-1 receptor-associated kinase 1 (IRAK1) [31-34].

Recent studies have shown that host miRNAs can inhibit viral replication by directly targeting viral genomic RNA, which has been regarded as a new mechanism of host antiviral defense [35-40] or a new way to change the viral life cycle [41-43]. In addition, viruses themselves can also encode miRNAs. These virus-derived miRNAs not only can modulate viral life cycle by directly regulating expression of viral self-genes or host-genes [44-46], but also can target host immune genes to help the virus to evade the immune response $[47,48]$. Unexpectedly, miR-122, a liver specific miRNA, promotes hepatitis $\mathrm{C}$ virus (HCV) replication by interacting with the $5^{\prime}$ untranslated region (UTR) of the viral genomic RNA [49]. So far, there are two major mechanisms that can explain why miR-122 facilitates HCV replication. First, miR-122 promotes the stabilization of HCV RNA [5052]; Second, miR-122 enhances translation of hepatitis $C$ virus RNA [53-55]. However, the phenomenon that miRNAs facilitate viral replication by direct interaction with viral RNAs is quite rare. Also, it has been proposed that the liver-specific miR-122 may contribute to $\mathrm{HCV}$ liver tropism at the level of translation [53]. Indeed, results from many studies support this hypothesis and demonstrate that miR-122 is a key determinant for HCV tropism [56-58]. Therefore, cellular miRNAs has probably exerted a significant influence on viral evolution.

\section{Identified functions of host miRNAs in PRRSV infection and replication}

PRRSV is a positive-sense single-stranded RNA virus and the viral genomic RNA has a long UTR in the downstream of opening reading frame $1 \mathrm{ab}$. In animals, most target mRNAs studied to date are regulated through 3' UTR interactions [11]. Therefore, it has been proposed that the long UTR might provide many targets for host miRNAs [5]. Indeed, many miRNA targets were predicted through computational approaches in a recent study, in which miR-181 was identified as a strong inhibitor of type 2 PRRSV [5]. Importantly, the miR-181 target region in the downstream region of open reading frame 4 of the viral genomic RNA was found to be highly conserved among type 2 PRRSV strains. Experimental studies demonstrated transfection of miR-181 mimics could strongly inhibit PRRSV replication through direct targeting of the viral genomic RNA in porcine alveolar macrophages (PAMs) or Marc-145 cells (a subclone of the monkey kidney cell line MA-104), suggesting that host miRNAs can interact with PRRSV RNAs, leading to the inhibition of viral replication [5]. Moreover, animal experiments provided direct evidence that miR-181 could repress PRRSV replication in vivo and that therapeutic miR-181 delivery could relieve the severity of symptoms in pigs infected with the highly pathogenic PRRSV strain and the treated pigs survived longer [5]. Taken together, these findings show that miR-181 is indeed a strong inhibitor of PRRSV.

In a subsequent study [6], we found that in addition to directly targeting the PRRSV genome, miR-181 was also confirmed to suppress PRRSV infection by downregulating its receptor, CD163. A previous study showed that the enhanced susceptibility of cultured peripheral blood monocytes (BMo) to PRRSV is coordinated well with the increased CD163 expression [59]. Interestingly, when CD163 mRNA was significantly upregulated in cultured BMo, miR-181 expression level was found to decrease gradually with or without L929 cell culture supernatants [6]. These results imply an inverse correlation between the expressions of miR-181 and CD163 expression during monocyte-macrophage differentiation. Application of a luciferase reporter system and an RNA-induced silencing complex immunoprecipitation assay confirmed that miR-181 can downregulate CD163 expression through directly targeting its mRNA [6]. Interestingly, results from construction of miR-181 target site mutated PRRSV demonstrated that miR-181 remarkably blocked both wild-type PRRSV and mutated PRRSV entry into PAMs at early times, but could only impair wild-type PRRSV replication at late times. Therefore, miR-181 may have a significant impact on PRRSV infection and replication in vivo.

Regulation of immune response by cellular miRNAs is an important issue. A recent published study showed that cellular miR-23 inhibits PRRSV replication by directly targeting PRRSV RNA and possibly by upregulating type I interferon [7]. In this study, two other miRNAs including miR-378 and miR-305 were also confirmed to inhibit PRRSV replication by directly targeting PRRSV RNA [7]. Moreover, the predicted target sites of miR-23, miR-378, and miR-505 are highly conserved (over 98\%, 96\% and $95 \%$, respectively) in type 2 PRRSV strains, suggesting these miRNAs may fundamentally regulate PRRSV replication. Interestingly, overexpression of miR-23 ren- 
dered the cells more competent in IFN- $\alpha$ and IFN- $\beta$ expression during PRRSV infection when compared with controls, but overexpression of miR-505 or miR-378 did not increase type I IFN expression [7]. More importantly, miR-23 did not mediate the activation of IFNs in the absence of PRRSV infection, suggesting that the activation of IFNs by miR-23 requires other signaling activated by PRRSV. Indeed, IRF3/IRF7 signaling was eventually confirmed to be critical for miR-23-mediated induction of IFNs during PRRSV infection [7]. As discussed above, some miRNAs can be induced by viral infection or TLR activation. Unexpectedly, PRRSV infection does not induce miR-23 and miR-505, but suppresses the expressions of poly(I:C)-induced miR-23 and miR-505. This antagonism might be a strategy for PRRSV to evade the miRNA-mediated immune response induced in TLR3 pathway.

\section{Are cellular miRNAs associated with PRRSV viral tropism?}

Although several miRNAs have been identified to inhibit PRRSV infection or replication, the question has to be asked: what are the roles of these miRNAs in PRRSV natural infection? In fact, the hypothesis that host miRNAs may have had a significant effect on viral evolution and may continue to influence the in vivo tissue tropism of viruses has been proposed in 2006 [60]. In its natural host, PRRSV has a narrow tropism for cells of the monocyte-macrophage lineage such as PAMs and subsets of macrophages in lymphoid tissues, but freshly isolated peripheral BMo and peritoneal macrophages are refractory to PRRSV infection
$[61,62]$. One of our previous studies showed that the expression of miR-181 and some other miRNAs negatively correlated with PRRSV infectivity in different cells and tissues [5]. For example, miR-181 was expressed at a much higher level in peripheral BMo and peritoneal macrophages than in PAMs (about 10- and 42-fold higher than that in PAMs, respectively), and the total cumulative expression of miRNAs which could potentially target PRRSV in peripheral BMo and peritoneal macrophages was also higher than that in PAMs [5]. Likewise in tissues, PRRSV-targeting miRNAs, such as miR-181 and miR338 , were abundant in the brain at a level that was about 5 times higher than that in the lungs, whereas virus load in the lungs was about 100 times higher than in the brain [5]. Therefore we postulate that the cellular miRNAs identified in that work may be associated with PRRSV tropism for different cells or tissues based on the role of cellular miRNAs in controlling virus replication. Importantly, miR181 has been confirmed to regulate CD163 in blood monocytes to determine PRRSV infection [6], suggesting that miR-181 may further influence PRRSV tropism also by direct targeting of CD163 mRNA. Besides, blockage of the endogenously expressed miR-23 significantly enhanced PRRSV replication, suggesting that endogenously miRNAs may control PRRSV replication during the whole infection process [7]. In addition, cellular miRNAs such as miR-23 may not only control PRRSV replication by direct targeting viral genomic RNA, but also inhibit its replication by inducing type I interferon. Thus, we propose that cellular miRNAs like miR-181 and miR-23 are likely to participate in the determination of cell or tissue tropism of PRRSV through targeting viral transcripts or viral receptors, or regulating antiviral immune response (Fig. 2).

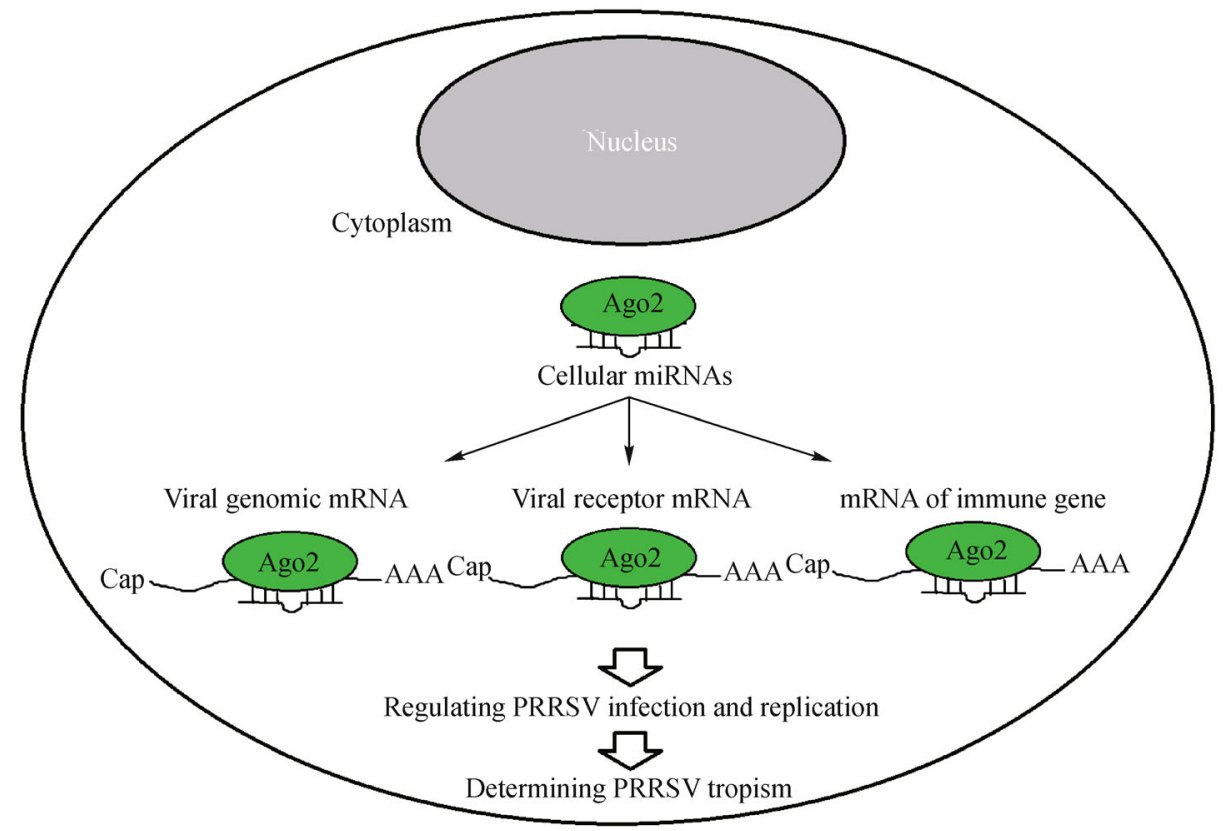

Fig. 2 A model of cellular miRNAs determining PRRSV tropism 


\section{Conclusions}

The study of miRNAs has contributed to the understanding of the regulation of viral infection. The targeting of viral transcripts and receptors, and induction of antiviral immune response by host miRNAs highlight the important role of miRNAs in PRRSV infection and also reveal mechanisms by which miRNAs could regulate tropism of PRRSV. Although much data have been obtained on the inhibition of PRRSV infection by miRNAs, direct functional data showing the exact effects of miRNAs on the tropism of PRRSV are still required. Specifically, the investigation of the function of miRNAs in other lesspermissive primary cells is needed to confirm their function in viral tropism.

Acknowledgements The support of the State Key Laboratory of Agrobiotechnology (2010SKLAB06-1 and 2012SKLAB01-6) and the Research Fund for the Doctoral Program of Higher Education of China (20130008110028) is gratefully acknowledged.

Compliance with ethics guidelines Xuekun Guo and Wenhai Feng declare that they have no conflict of interest or financial conflicts to disclose.

This article does not contain any studies with human or animal subjects performed by any of the authors.

\section{References}

1. Kimman T G, Cornelissen L A, Moormann R J, Rebel J M, Stockhofe-Zurwieden N. Challenges for porcine reproductive and respiratory syndrome virus (PRRSV) vaccinology. Vaccine, 2009, 27(28): 3704-3718

2. Bartel D P. MicroRNAs: target recognition and regulatory functions. Cell, 2009, 136(2): 215-233

3. Carthew R W, Sontheimer E J. Origins and mechanisms of miRNAs and siRNAs. Cell, 2009, 136(4): 642-655

4. Krol J, Loedige I, Filipowicz W. The widespread regulation of microRNA biogenesis, function and decay. Nature Reviews Genetics, 2010, 11(9): 597-610

5. Guo X K, Zhang Q, Gao L, Li N, Chen X X, Feng W H. Increasing expression of microRNA 181 inhibits porcine reproductive and respiratory syndrome virus replication and has implications for controlling virus infection. Journal of Virology, 2013, 87(2): 11591171

6. Gao L, Guo X K, Wang L, Zhang Q, Li N, Chen X X, Wang Y, Feng W H. MicroRNA 181 suppresses porcine reproductive and respiratory syndrome virus (PRRSV) infection by targeting PRRSV receptor CD163. Journal of Virology, 2013, 87(15): 8808-8812

7. Zhang Q, Guo X K, Gao L, Huang C, Li N, Jia X, Liu W, Feng W H. MicroRNA-23 inhibits PRRSV replication by directly targeting PRRSV RNA and possibly by upregulating type I interferons. Virology, 2014, 450-451: 182-195

8. Hicks J A, Yoo D, Liu H C. Characterization of the microRNAome in porcine reproductive and respiratory syndrome virus infected macrophages. PLoS ONE, 2013, 8(12): e82054
9. Kim V N, Han J, Siomi M C. Biogenesis of small RNAs in animals. Nature Reviews Molecular Cell Biology, 2009, 10(2): 126-139

10. Kim V N. MicroRNA biogenesis: coordinated cropping and dicing. Nature Reviews Molecular Cell Biology, 2005, 6(5): 376-385

11. Pasquinelli A E. MicroRNAs and their targets: recognition, regulation and an emerging reciprocal relationship. Nature Reviews Genetics, 2012, 13(4): 271-282

12. Huntzinger E, Izaurralde E. Gene silencing by microRNAs: contributions of translational repression and mRNA decay. Nature Reviews Genetics, 2011, 12(2): 99-110

13. Doench J G, Sharp P A. Specificity of microRNA target selection in translational repression. Genes \& Development, 2004, 18(5): 504511

14. Reinhart B J, Slack F J, Basson M, Pasquinelli A E, Bettinger J C, Rougvie A E, Horvitz H R, Ruvkun G. The 21-nucleotide let-7 RNA regulates developmental timing in Caenorhabditis elegans. Nature, 2000, 403(6772): 901-906

15. Slack F J, Basson M, Liu Z, Ambros V, Horvitz H R, Ruvkun G. The lin-41 RBCC gene acts in the C. elegans heterochronic pathway between the let-7 regulatory RNA and the LIN-29 transcription factor. Molecular Cell, 2000, 5(4): 659-669

16. Vella M C, Choi E Y, Lin S Y, Reinert K, Slack F J. The C. elegans microRNA let-7 binds to imperfect let-7 complementary sites from the lin-41 3' UTR. Genes \& Development, 2004, 18(2): 132-137

17. Shin C, Nam J W, Farh K K, Chiang H R, Shkumatava A, Bartel D P. Expanding the microRNA targeting code: functional sites with centered pairing. Molecular Cell, 2010, 38(6): 789-802

18. Rigoutsos I. New tricks for animal microRNAS: targeting of amino acid coding regions at conserved and nonconserved sites. Cancer Research, 2009, 69(8): 3245-3248

19. Tay Y, Zhang J, Thomson A M, Lim B, Rigoutsos I. MicroRNAs to Nanog, Oct4 and Sox 2 coding regions modulate embryonic stem cell differentiation. Nature, 2008, 455(7216): 1124-1128

20. Akira S, Uematsu S, Takeuchi O. Pathogen recognition and innate immunity. Cell, 2006, 124(4): 783-801

21. Kawai T, Akira S. The role of pattern-recognition receptors in innate immunity: update on Toll-like receptors. Nature Immunology, 2010, 11(5): 373-384

22. O'Neill L A, Sheedy F J, McCoy C E. MicroRNAs: the fine-tuners of Toll-like receptor signalling. Nature Reviews Immunology, 2011, 11(3): 163-175

23. O'Connell R M, Rao D S, Chaudhuri A A, Baltimore D. Physiological and pathological roles for microRNAs in the immune system. Nature Reviews Immunology, 2010, 10(2): 111-122

24. O'Connell R M, Taganov K D, Boldin M P, Cheng G, Baltimore D. MicroRNA-155 is induced during the macrophage inflammatory response. Proceedings of the National Academy of Sciences of the United States of America, 2007, 104(5): 1604-1609

25. Taganov K D, Boldin M P, Chang K J, Baltimore D. NF- $\kappa B-$ dependent induction of microRNA miR-146, an inhibitor targeted to signaling proteins of innate immune responses. Proceedings of the National Academy of Sciences of the United States of America, 2006, 103(33): 12481-12486

26. Thai T H, Calado D P, Casola S, Ansel K M, Xiao C, Xue Y, Murphy A, Frendewey D, Valenzuela D, Kutok J L, SchmidtSupprian M, Rajewsky N, Yancopoulos G, Rao A, Rajewsky K. 
Regulation of the germinal center response by microRNA-155. Science, 2007, 316(5824): 604-608

27. O’Connell R M, Chaudhuri A A, Rao D S, Baltimore D. Inositol phosphatase SHIP1 is a primary target of miR-155. Proceedings of the National Academy of Sciences of the United States of America, 2009, 106(17): 7113-7118

28. Androulidaki A, Iliopoulos D, Arranz A, Doxaki C, Schworer S, Zacharioudaki V, Margioris A N, Tsichlis P N, Tsatsanis C. The kinase Akt1 controls macrophage response to lipopolysaccharide by regulating microRNAs. Immunity, 2009, 31(2): 220-231

29. Wang P, Hou J, Lin L, Wang C, Liu X, Li D, Ma F, Wang Z, Cao X. Inducible microRNA-155 feedback promotes type I IFN signaling in antiviral innate immunity by targeting suppressor of cytokine signaling 1. Journal of Immunology, 2010, 185(10): 6226-6233

30. Hou J, Wang P, Lin L, Liu X, Ma F, An H, Wang Z, Cao X. MicroRNA-146a feedback inhibits RIG-I-dependent Type I IFN production in macrophages by targeting TRAF6, IRAK1, and IRAK2. Journal of Immunology, 2009, 183(3): 2150-2158

31. Sheedy F J, Palsson-McDermott E, Hennessy E J, Martin C, O'Leary J J, Ruan Q, Johnson D S, Chen Y, O'Neill L A. Negative regulation of TLR4 via targeting of the proinflammatory tumor suppressor PDCD4 by the microRNA miR-21. Nature Immunology, 2010, 11(2): 141-147

32. Chen Y, Chen J, Wang H, Shi J, Wu K, Liu S, Liu Y, Wu J. HCVinduced miR-21 contributes to evasion of host immune system by targeting MyD88 and IRAK1. PLoS Pathogens, 2013, 9(4): e1003248

33. Cohen T S, Prince A S. Bacterial pathogens activate a common inflammatory pathway through IFN $\lambda$ regulation of PDCD4. PLoS Pathogens, 2013, 9(10): e1003682

34. Moschos S A, Williams A E, Perry M M, Birrell M A, Belvisi M G, Lindsay $\mathrm{M}$ A. Expression profiling in vivo demonstrates rapid changes in lung microRNA levels following lipopolysaccharideinduced inflammation but not in the anti-inflammatory action of glucocorticoids. BMC Genomics, 2007, 8(1): 240

35. Pedersen I M, Cheng G, Wieland S, Volinia S, Croce C M, Chisari F $\mathrm{V}$, David M. Interferon modulation of cellular microRNAs as an antiviral mechanism. Nature, 2007, 449(7164): 919-922

36. Lecellier C H, Dunoyer P, Arar K, Lehmann-Che J, Eyquem S, Himber C, Saïb A, Voinnet O. A cellular microRNA mediates antiviral defense in human cells. Science, 2005, 308(5721): 557560

37. Li H W, Ding S W. Antiviral silencing in animals. FEBS Letters, 2005, 579(26): 5965-5973

38. Nathans R, Chu C Y, Serquina A K, Lu C C, Cao H, Rana T M. Cellular microRNA and $\mathrm{P}$ bodies modulate host-HIV-1 interactions. Molecular Cell, 2009, 34(6): 696-709

39. Otsuka M, Jing Q, Georgel P, New L, Chen J, Mols J, Kang Y J, Jiang Z, Du X, Cook R, Das S C, Pattnaik A K, Beutler B, Han J. Hypersusceptibility to vesicular stomatitis virus infection in Dicer1deficient mice is due to impaired miR24 and miR93 expression. Immunity, 2007, 27(1): 123-134

40. Song L, Liu H, Gao S, Jiang W, Huang W. Cellular microRNAs inhibit replication of the H1N1 influenza A virus in infected cells. Journal of Virology, 2010, 84(17): 8849-8860

41. Gottwein E, Cullen B R. Viral and cellular microRNAs as determinants of viral pathogenesis and immunity. Cell Host \& Microbe, 2008, 3(6): 375-387

42. Chen Y, Shen A, Rider P J, Yu Y, Wu K, Mu Y, Hao Q, Liu Y, Gong $\mathrm{H}, \mathrm{Zhu}$ Y, Liu F, Wu J. A liver-specific microRNA binds to a highly conserved RNA sequence of hepatitis $B$ virus and negatively regulates viral gene expression and replication. FASEB Journal, 2011, 25(12): 4511-4521

43. Huang J, Wang F, Argyris E, Chen K, Liang Z, Tian H, Huang W, Squires K, Verlinghieri G, Zhang H. Cellular microRNAs contribute to HIV-1 latency in resting primary $\mathrm{CD}^{+}{ }^{+} \mathrm{T}$ lymphocytes. Nature Medicine, 2007, 13(10): 1241-1247

44. Umbach J L, Kramer M F, Jurak I, Karnowski H W, Coen D M, Cullen B R. MicroRNAs expressed by herpes simplex virus 1 during latent infection regulate viral mRNAs. Nature, 2008, 454(7205): 780-783

45. Pfeffer S, Zavolan M, Grässer F A, Chien M, Russo J J, Ju J, John B, Enright A J, Marks D, Sander C, Tuschl T. Identification of virusencoded microRNAs. Science, 2004, 304(5671): 734-736

46. Lu C C, Li Z, Chu C Y, Feng J, Feng J, Sun R, Rana T M. MicroRNAs encoded by Kaposi's sarcoma-associated herpesvirus regulate viral life cycle. EMBO Reports, 2010, 11(10): 784-790

47. Kim S, Lee S, Shin J, Kim Y, Evnouchidou I, Kim D, Kim Y K, Kim Y E, Ahn J H, Riddell S R, Stratikos E, Kim V N, Ahn K. Human cytomegalovirus microRNA miR-US4-1 inhibits $\mathrm{CD}^{+} \mathrm{T}$ cell responses by targeting the aminopeptidase ERAP1. Nature Immunology, 2011, 12(10): 984-991

48. Nachmani D, Lankry D, Wolf D G, Mandelboim O. The human cytomegalovirus microRNA miR-UL112 acts synergistically with a cellular microRNA to escape immune elimination. Nature Immunology, 2010, 11(9): 806-813

49. Jopling C L, Yi M, Lancaster A M, Lemon S M, Sarnow P. modulation of hepatitis $\mathrm{C}$ virus RNA abundance by a liver-specific microRNA. Science, 2005, 309(5740): 1577-1581

50. Mortimer S A, Doudna J A. Unconventional miR-122 binding stabilizes the HCV genome by forming a trimolecular RNA structure. Nucleic Acids Research, 2013, 41(7): 4230-4240

51. Shimakami T, Yamane D, Jangra R K, Kempf B J, Spaniel C, Barton D J, Lemon S M. Stabilization of hepatitis C virus RNA by an Ago2miR-122 complex. Proceedings of the National Academy of Sciences of the United States of America, 2012, 109(3): 941-946

52. Shimakami T, Yamane D, Welsch C, Hensley L, Jangra R K, Lemon $\mathrm{S} \mathrm{M}$. Base pairing between hepatitis $\mathrm{C}$ virus RNA and microRNA $1223^{\prime}$ of its seed sequence is essential for genome stabilization and production of infectious virus. Journal of Virology, 2012, 86(13): 7372-7383

53. Henke J I, Goergen D, Zheng J, Song Y, Schüttler C G, Fehr C, Jünemann C, Niepmann M. microRNA-122 stimulates translation of hepatitis C virus RNA. EMBO Journal, 2008, 27(24): 3300-3310

54. Jangra R K, Yi M, Lemon S M. Regulation of hepatitis C virus translation and infectious virus production by the microRNA miR122. Journal of Virology, 2010, 84(13): 6615-6625

55. Niepmann M. Activation of hepatitis $\mathrm{C}$ virus translation by a liverspecific microRNA. Cell Cycle, 2009, 8(10): 1473-1477

56. Fukuhara T, Kambara H, Shiokawa M, Ono C, Katoh H, Morita E, Okuzaki D, Maehara Y, Koike K, Matsuura Y. Expression of microRNA miR-122 facilitates an efficient replication in nonhepatic 
cells upon infection with hepatitis C virus. Journal of Virology, 2012, 86(15): 7918-7933

57. Schwartz R E, Trehan K, Andrus L, Sheahan T P, Ploss A, Duncan S A, Rice C M, Bhatia S N. Modeling hepatitis $\mathrm{C}$ virus infection using human induced pluripotent stem cells. Proceedings of the National Academy of Sciences of the United States of America, 2012, 109(7): 2544-2548

58. Wu X, Robotham J M, Lee E, Dalton S, Kneteman N M, Gilbert D $\mathrm{M}$, Tang H. Productive hepatitis $\mathrm{C}$ virus infection of stem cellderived hepatocytes reveals a critical transition to viral permissiveness during differentiation. PLoS Pathogens, 2012, 8(4): e1002617

59. Wang L, Zhang H, Suo X, Zheng S, Feng W H. Increase of CD163 but not sialoadhesin on cultured peripheral blood monocytes is coordinated with enhanced susceptibility to porcine reproductive and respiratory syndrome virus infection. Veterinary Immunology and Immunopathology, 2011, 141(3-4): 209-220

60. Cullen B R. Viruses and microRNAs. Nature Genetics, 2006, 38 (Suppl): S25-S30

61. Duan X, Nauwynck H J, Pensaert M B. Effects of origin and state of differentiation and activation of monocytes/macrophages on their susceptibility to porcine reproductive and respiratory syndrome virus (PRRSV). Archives of Virology, 1997, 142(12): 2483-2497

62. Duan X, Nauwynck H J, Pensaert M B. Virus quantification and identification of cellular targets in the lungs and lymphoid tissues of pigs at different time intervals after inoculation with porcine reproductive and respiratory syndrome virus (PRRSV). Veterinary Microbiology, 1997, 56(1-2): 9-19 